\title{
InDRC 2015 - The Tradition of Drosophila Research in India has Attained a Critical Mass
}

\author{
KRISHANU RAY* \\ Department of Biological Sciences, TIFR, Mumbai 400 005, India
}

Drosophila, the dew flies, are so named because of the buzz of these nearly invisible insects encountered during a walk on the early morning dew covered grass. They feed on fermented organic matter, fruits and vegetables, and, therefore are also called fruit flies. They are found almost all over the tropics and subtropics. A chance encounter followed by a systematic research by pioneers, Thomas Hunt Morgan and his colleagues, in the 'Fly Room' of Columbia University, during the early $20^{\text {th }}$ Century has made a particular species of the Mediterranean fruit flies, Drosophila melanogaster, the darling of nearly 7000 laboratories worldwide. These flies are now literally buzzing around the world, jet-setting the pace of biological research in a broad variety of wideranging topics - evolution, genetics, cell and developmental biology, neurobiology and many others. The underlying causes of a variety of human diseases are also studied in these insects to fast-track the discovery of underlying factors and possible cures.

From the very beginning, Drosophila researchers have broken many new grounds. It was used for the first time to establish that genes, the units of inheritance, are physically located on the chromosomes. The study of salivary gland chromosomes initiated by Morgan's associate, T S Painter (Painter, 1934), and continued by many others, led to a large number of discoveries relating to organization of genes and regulation of their expression. Such studies were particularly facilitated by some extraordinarily detailed and accurate drawings of polytene chromosomes of Drosophila melanogaster made by Bridges in the late 1930s. Drosophila was also used to test the theory of evolution by abrupt changes through mutagenesis. The early discovery of spontaneously occurring white eyed flies, and other eye and body color mutants, as well as the Mendelian propagation of these traits to the progeny, measured and reestablished the theory of genetics on a firm footing. Further, research on Drosophila provided the first experimental leads to the genes that control development and pattern formation (Nusslein-Volhard and Wieschaus, 1980; Lewis 1998). Interestingly, Morgan, the father of modern genetics, did not initially believe in the chromosomal basis of inheritance or natural selection while the classical embryologists in the first half of 20th century did not believe in genes having any role in embryonic development (Gilbert, 1978). Eventually, the research using flies has spread into every nook and corner of biology because of the egalitarian research fraternity, large-scale development and free sharing of mutated/engineered fly stocks and the relative ease with which a fly lab can be set up in a small place.

On a chilly December 2015 afternoon, a host of fly pushers, a nickname for Drosophila researchers, descended from different parts of the country on the serene campus of Indian Institute of TechnologyKanpur and buzzed around for three days. It was the occasion of the biennial Indian Drosophila Research Conference (InDRC 2015). Nearly 50 faculty members and about 170 students assembled in the Outreach Auditorium for three days. They deliberated on matters related to Development and Disease, Cellular morphogenesis and molecular techniques. The meeting started with a keynote address by $\mathrm{S} \mathrm{C}$ Lakhotia outlining the chronology and the academic lineages of Drosophila researchers in India. The tradition of Drosophila-based research in India is quite old. It started almost at the same time in Kolkata and Mysore and later on spread to other parts of the

*Author for Correspondence: E-mail: krishanu@tifr.res.in 
country. The lineages of early Drosophila researchers in India can be traced to the real pioneers of Drosophila research (Fig. 1). The meeting reached a crescendo on the last day with the presentation of N G Prasad, describing the evolution of immune system through laboratory-scale experiments using Drosophila. From many different perspectives, this was an exciting amalgamation of research ideas, as far apart as evolution and molecular immunology, in a single experimental paradigm. It is possible only using Drosophila. Therefore, it was little wonder that the talk was judged as the best of the conference by the audience. It is an appreciation of the reach and potential of experimental research possible in fruit flies.

In the beginning, the fly community's research in India was restricted to Cytogenetics and Evolution. Over the decades, it has spread to every other aspect including Neurogenetics, Behaviour, Molecular Genetics, Cell and Developmental Biology, Immunology etc. The tradition of annual meetings of the Indian Drosophila researchers started at the Mysore University in the late 1980s, and it continued uninterrupted for several years. However, the momentum could not be sustained. A new effort was made when the $6^{\text {th }}$ Indian Drosophila Meeting was organized during November 19-20, 2010 at the University of Mysore after a long gap. It was further revitalized with a brief meeting at Banaras Hindu University, Varanasi, in March 2014 when nearly 42 different faculty members assembled and pledged to rekindle the fly community in India (Ray, 2014).

The Kanpur meeting, organized during December 20-23, 2015, was continuation of the revised biennial series format agreed at the BHU meeting. It was attended by about fifty faculty members and



Fig. 1: The lineages and chronology of early pioneers of Drosophila research in India (provided by S C Lakhotia, BHU, Varanasi) 
about 170 students and post-docs from across India. It was formally named InDRC, an acronym for Indian Drosophila Research Conference, and the members resolved to continue meeting every two years.

The speakers discussed unpublished results from ongoing research in their laboratories. The topics were very diverse, ranging from collective cell behavior, oncogenesis, stem cells, RNA biology, cell signaling, neurobiology, muscle development and many more. All through the meeting, the audience remained engaged by seeking clarifications, asking questions and debating the conclusions. Another important feature of the meeting was the technical sessions where the state of the art techniques developed by Drosophila-based researchers and the advanced microscopy tools useful for fly research were discussed by experts. Active engagement by the participating student community was one of the key highlights of the meeting. The poster sessions were well attended, and it was felt that more time was needed to do justice to the excellent caliber of the posters presented at the meeting. The schedule and

\section{References}

Gilbert S F (1978) The embryological origins of the gene theory J History Biol 11 307-351

Lewis E B (1998) The bithorax complex: the first fifty years Int J Dev Biol 42 403-415

Nusslein-Volhard C and Wieschaus E (1980) Mutations affecting segment number and polarity in Drosophila Nature 287 795-801 abstracts of the meeting are available online (www.devbioindia.org).

The organism-centric conferences have a definite advantage in disseminating usages of advanced tools amongst the community. The American Drosophila Meetings, organized under auspices of The Genetics Society of America, have been serving this purpose most efficiently. Those meetings have helped to build consortia amongst the researchers and brought in people with diverse capabilities on one platform. InDRC 2015 reassured that the fly community in India has reached the criticality. There was a competition for hosting the next biennial meeting, InDRC 2017. The venue for the next meeting, selected by the participating members, would be the Indian Institute of Science Education and Research, Bhopal.

\section{Acknowledgment}

The author sincerely thanks Professor S C Lakhotia, Cytogenetics Laboratory, Banaras Hindu University, Varanasi for critical reading and helpful suggestions.

Painter T S (1934) Salivary chromosomes and the attack on the gene $J$ Heredity 25 465-476

Ray K (2014) Research using the fruit fly Drosophila - a great conduit of knowledge in modern biology - gained traction in India Proc Indian Natn Sci Acad 80 187-191. 